\title{
Study on Bifurcation and Chaos in Boost Converter Based on Energy Balance Model
}

\author{
Quanmin NIU, Zhizhong JU \\ Department of Information Countermeasure, Air Force Radar Academy, AFRA, Wuhan, China \\ Email:nqm1@tom.com
}

\begin{abstract}
Based on boost converter operating in discontinuous mode, this paper proposes an energy balance model (EBM) for analyzing bifurcation and chaos phenomena of capacitor energy and output voltage when the converter parameter is varying. It is found that the capacitor energy and output voltage dynamic behaviors exhibit the typical period-doubling route to chaos by increasing the feedback gain constant $K$ of proportional controller. The accurate position of the first bifurcation point and the iterative diagram of the capacitor energy with every $K$ can be derived from EBM. Finally, the underlying causes for bifurcations and chaos of a general class of nonlinear systems such as power converters are analyzed from the energy balance viewpoint. Comparing with the discrete iterative model, EBM is simple and high accuracy. This model can be easily developed on the nonlinear study of the other converters.
\end{abstract}

Keywords: power converter, nonlinear, bifurcation, chaos, energy balance model

\section{Introduction}

The bifurcation and chaos phenomena appeared in power system have becoming a focus subject at present. It is found that basic DC/DC converters exhibit bifurcation and chaos phenomena as well as parallel-connected DC/DC converters and PFC system. There are no unified methods in researching nonlinear power system. In the appeared literatures, the averaged model and the sampling data model are usually adopted in nonlinear analysis for DC/DC converters [1][2]. But the averaged model neglects the dynamic characteristic of the system at high frequency, and only can be used for analyzing the dynamic behavior at low frequency. This model has limitations as follows. Firstly, the dependence on initial condition for system dynamic behavior is neglected in small signal analysis and it can't predict dynamic behavior when the converters work in the saturated mode. Secondly, this model can't predict the instability of system under the fast-scale condition [3]. According to the periodic working characteristic of power system, the sampling data modeling method build the relationship be- tween state variable at present sampling time instant and state variable at next sampling time instant. Based on this idea, four models named stroboscopic map model, synchronous switching map model, asynchronous switching map model and general two-by-two switching map model are given in [2] according to the different sampling time instant. These models are used to analyze nonlinear phenomena such as bifurcation and chaos. But some shortcomings exist in these models. For example, it is difficult to get accurate analytic models as the duty ratio of control pulses is the nonlinear function of state variables. If we neglect nonlinear effect on system transfer matrix, big errors and much amount of calculation will bring about. In order to improve the accuracy of simulation results, this paper proposes an energy balance model (EBM) for boost converter. According to energy balance principle, EBM was established and the dynamic behavior of the capacitor energy state was investigated. From the bifurcation diagrams of capacitor energy state and output voltage, EBM presented in this paper is more accurate than the stroboscopic map model presented in [4]. Furthermore, 


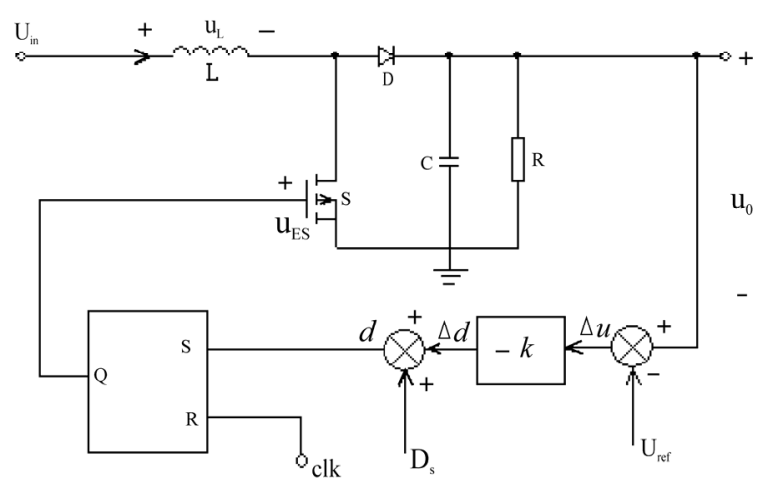

Figure 1. Schematic circuit diagram of closed-loop boost converter

energy bifurcation mechanism for power converters can be found from EBM.

\section{Building Energy Balance Model}

The circuit diagram of the closed-loop boost converter is shown in Figure 1. Suppose that boost converter works in DCM. The feedback gain constant of voltage amplifier is $k$ and error signal $\Delta u$ is amplified through the voltage amplifier. The amplified error voltage compares with saw-tooth wave signal and produces variable duty cycle control pulse. The variable duty cycle denotes $\Delta d$ in Figure 1. The output voltage is regulated by changing $\Delta d$ when input voltage and output load is fluctuating.

In one switching period $[n T,(n+1) T]$, the converter satisfies energy balance condition

$$
\Delta E_{\text {in }}=\Delta E_{R}+\Delta E_{C}+\Delta E_{L},
$$

where $\Delta E_{\text {in }} 、 \Delta E_{R} 、 \Delta E_{L} 、 \Delta E_{c}$ denote the energy supplied by input power, the energy consumed on resistance load, the storage energy in inductor and the storage energy in capacitor respectively in one switching period. In DCM converter, the inductor current always starts from zero, i.e., $\Delta E_{L}=0$, so the energy balance formula is

$$
\Delta E_{\text {in }}=\Delta E_{R}+\Delta E_{c} .
$$

The current flowing out from input power equals to the current flowing into inductor in a switching period as shown in Figure 2.

According to Figure 2, we can write

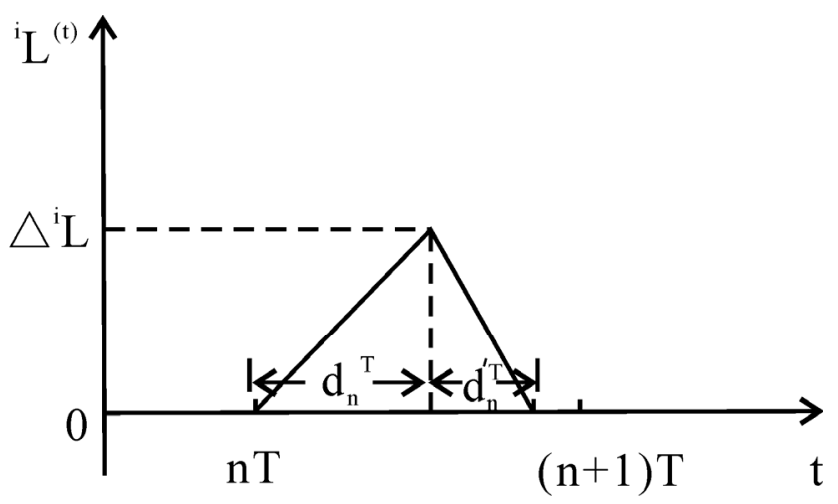

Figure 2. Inductor current waveform

$$
\Delta i_{L}=\frac{U_{i n}}{L} d_{n} T=\frac{U_{r e f}-U_{i n}}{L} d^{\prime}{ }_{n} T .
$$

So, we obtain

$$
d_{n}^{\prime}=\frac{U_{i n}}{U_{r e f}-U_{i n}} d_{n} .
$$

$\Delta E_{\text {in }}$ can be written as

$$
\begin{aligned}
& \Delta E_{\text {in }}=U_{\text {in }} \int_{n T}^{(n+1) T} i_{L}(t) d t \approx \frac{1}{2} \Delta i_{L}\left(d_{n}+d_{n}^{\prime}\right) T U_{i n} \\
& =\frac{U_{\text {in }}{ }^{2} T^{2}}{2 L} \frac{U_{r e f}}{U_{r e f}-U_{\text {in }}} d_{n}{ }^{2}
\end{aligned}
$$

The energy consumed by load is

$$
\begin{aligned}
& \Delta E_{R}=\frac{1}{R} \int_{n T}^{(n+1) T} u_{c}^{2}(t) d t \approx \frac{T}{2}\left[\frac{u_{c, n T}^{2}}{R}+\frac{u_{c,(n+1) T}{ }^{2}}{R}\right] . \\
& =\frac{T}{R C}\left[E_{c, n T}+E_{c,(n+1) T}\right]
\end{aligned} .
$$

The storage energy in capacitor in one switching period is

$$
\Delta E_{c}=E_{c,(n+1) T}-E_{c, n T} .
$$

Substitute (6), (7) into (2), we obtain

$$
E_{c,(n+1) T}=K_{S} \cdot E_{c, n T}+\frac{1}{1+T / R C} \Delta E_{i n} .
$$

Substitute (5) into (8), we can build EBM of boost converter

$$
E_{c,(n+1) T}=K_{S} E_{c, n T}+A d_{n}^{2},
$$


where $K_{S}=\frac{1-T / R C}{1+T / R C}, A=\frac{1}{1+T / R C} \frac{U_{i n}{ }^{2} T^{2}}{2 L} \frac{U_{r e f}}{U_{r e f}-U_{i n}}$.

As shown in Figure 1, the duty cycle $d_{n}$ in the $n$th period is

$$
d_{n}=D_{S}-K\left(u_{n}-U_{r e f}\right),
$$

where $D_{s}$ is steady duty cycle, $u_{n}$ is sampling value of output voltage at the $n$th period.

After the converter enters into steady state, the closed-loop system should satisfy

$$
E_{c,(n+1) T}=E_{c, n T}=\frac{1}{2} C U_{r e f}^{2}=E_{r e f} .
$$

From (9) and (11), the steady duty cycle expression is

$$
D_{S}=\sqrt{\frac{1-K_{S}}{A} E_{r e f}} .
$$

For actual converter, $d_{n}$ should satisfy the following expression

$$
d_{n}=\left\{\begin{array}{c}
0 ; D_{S}-K\left(u_{n}-U_{r e f}\right)<0 \\
D_{S}-K\left(u_{n}-U_{r e f}\right) ; 0<D_{S}-K\left(u_{n}-U_{r e f}\right)<1 . \\
1 ; D_{S}-K\left(u_{n}-U_{r e f}\right)>1
\end{array}\right.
$$

\section{Results and Discussions}

\subsection{Diagrams of Storage Energy in Capacitor}

Based on (9) and (13),we can depict the sequence diagram of capacitor energy for boost converter operated in period 2 as shown in Figure 3. The circuit parameters is

$$
t=333.3 \mu \mathrm{s}, U_{\text {in }}=16 \mathrm{~V}, U_{\text {ref }}=25 \mathrm{~V}, L=208 \mu \mathrm{H}, \mathrm{C}=222 \mu \mathrm{F}, R=12.5 \Omega
$$

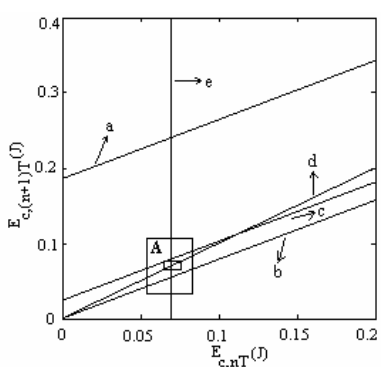

(a) $\mathrm{K}=0.1$ Period 2

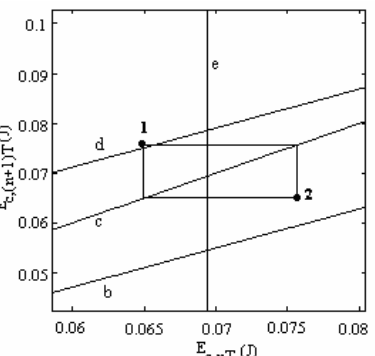

(b) Enlarge diagram of area $\mathrm{A}$ in (a)
Figure 3. Energy locus of capacitor in period 2
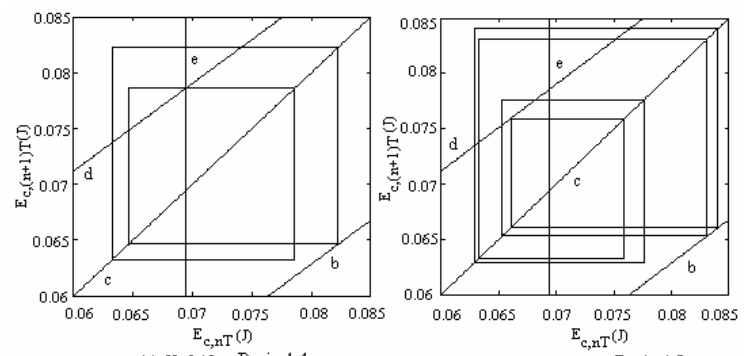

(a) $\mathrm{K}=0.12$ Period 4

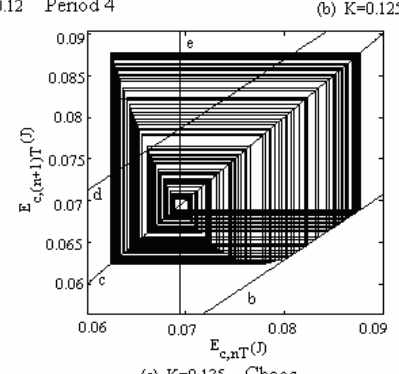

(c) $\mathrm{K}=0.135$ Chaos

Figure 4. Energy locus of capacitor in period 4, period 8 and chaos
The storage energy in other period oscillation is shown in Figure 4. The lines appeared in Figure 3 and Figure 4 defined as follows.

Substitute $d_{n}=1$ into (9), we can write

$$
E_{c,(n+1) T}=K_{S} E_{c, n T}+A
$$

Therefore, line $a$ is depicted from the above expression.

Likewise, substitute $d_{n}=0$ into (9), we obtain

$$
E_{c,(n+1) T}=K_{S} E_{c, n T}
$$

The line $b$ is depicted from (15).

Line $a$ and line $b$ are two parallel lines and show storage energy state in capacitor under two utmost conditions. For actual converter, duty cycle is limited and the energy locus is also limited between $a$ and $b$ If the energy locus touch with line $b$,it shows that the duty cycle of corresponding control pulse is zero, i.e., power switch has being turned-off.

When storage energy in capacitor is in equilibrium, the equation is expressed as the following

$$
E_{c,(n+1) T}=E_{c, n T} .
$$


Line $c$ is depicted from (16).

In CCM and critical mode, $d_{n}+d_{n}^{\prime}=1$, and in DCM, $d_{n}+d_{n}^{\prime}<1$.

From (4), we obtain the duty cycle of converter operating in critical mode.

$$
d_{n c}=\frac{U_{r e f}-U_{i n}}{U_{r e f}}
$$

So, the storage energy in capacitor can be depicted as line $d$. The corresponding equation can be written as

$$
E_{c,(n+1) T}=K_{S} E_{c, n T}+A\left(\frac{U_{r e f}-U_{i n}}{U_{r e f}}\right)^{2} .
$$

It is obvious that the converter will enter into CCM while energy locus lies above line $d$, and DCM while energy locus lies below line $d$.

Line $e$ denotes reference energy, the equation is

$$
E_{r e f}=\frac{1}{2} C U_{r e f}^{2}
$$

Figure 3(b) is the enlarge diagram of energy locus in period 2. As shown in Figure 3(b), energy state transforms between dot 1 and dot 2 while dot 1 lies in the left upper part of line $d$ and dot 2 lies in the right lower part of line $d$. This means that inductor current on $\operatorname{dot} 1$ works in CCM, and inductor current on dot 2 works in DCM. Because there is no intersection between energy locus and line $b$, it indicates that there are no skipped cycles in period 2 oscillation.

For the other periodic oscillation behavior, the operation characteristics of converter are comprehended by the energy locus diagrams as shown in Figure 4. For example, the operation characteristics in period 4 are described as follows. Inductor current is continuous in the first switching period; and discontinuous in the second switching period accompanying with skipped cycles. Furthermore, inductor current returns to be continuous in the third switching period; and discontinuous without skipped cycles in the fourth switching period. Such switching sequences make output voltage transform among four values. The same conclusion will be obtained by analyzing other energy locus in Figure 4(a) and Figure 4(b). However, from Figure 4(c) we can find that energy locus is much complex and has many intersections with line $b$. Meanwhile, many energy states lie on the top of line $d$. It shows that the corresponding control pulses sequences is very complex. The output of converter has entered into chaos. .

In general, we can acquire much operation information of converter from the diagram of capacitor energy locus. The energy locus of capacitor moves around the reference energy (line $e$ ). Therefore, the corresponding output voltage $u_{0}$ is fluctuating at $U_{r e f}$. The energy states on the top of line $d$ shows that converter operates in CCM and the energy states at the bottom of line $d$ shows that converter operates in DCM. The points where energy locus intersects with line $b$ indicate that converter operates with skipped cycles.

\subsection{Bifurcation Diagrams}

As shown in Figure 5, the bifurcation diagram of the capacitor energy is derived from (9) and (13) when the feedback gain constant $k$ of error voltage amplifier is varying. It is obvious that the capacitor energy exhibit the route from period-doubling to chaos by increasing $k$.

Assume that the converter is in steady state. $0<d_{n}<1$.

Substituting $d_{n}=D_{s}-K\left(u_{n}-U_{\text {ref }}\right)$ into (9) and using the following expressions

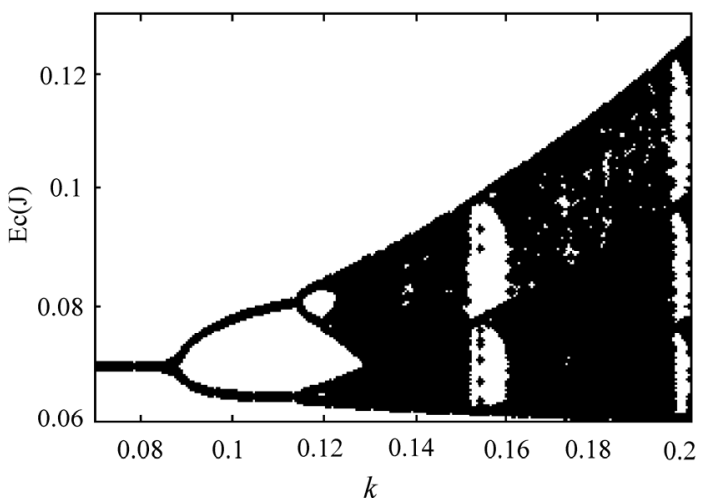

Figure 5. Bifurcation diagram of capacitor storage energy 


$$
\begin{gathered}
u_{n}=\sqrt{\frac{2 E_{c, n T}}{C}}, \\
U_{r e f}=\sqrt{\frac{2 E_{r e f}}{C}} .
\end{gathered}
$$

We can write

$$
E_{c,(n+1) T}=K_{S} E_{c, n T}+A\left[D_{S}-K\left(\sqrt{\frac{2 E_{c, n T}}{C}}-\sqrt{\frac{2 E_{r e f}}{C}}\right)\right]^{2} .
$$

It is obvious that (22) can be expanded with Taylor's series at the equilibrium point $E_{c, n T Q}=E_{r e f}$. If high-level items of Taylor's series are neglected, the equation can be written as

$$
\Delta E_{c,(n+1) T}=\lambda \cdot \Delta E_{c . n T}
$$

where $\lambda=\left.\frac{\partial E_{c,(n+1) T}}{\partial E_{c, n T}}\right|_{E_{c, n T Q}=E_{r e f}}=K_{S}-\frac{2 A D_{S} K}{\sqrt{2 C E_{r e f}}}$.

In the range of small signals, $\lambda$ can determine system stability. When $-1<\lambda<1$, system will be steady. The position of the first bifurcation point can be obtained at $\lambda=-1$

$$
K_{c}=\left(1+K_{S}\right) \frac{\sqrt{2 C E_{r e f}}}{2 A D_{S}}=0.09865
$$

Bifurcation diagrams of output voltage simulated by three models are shown as Figure 6. The diagram of Figure 6(a) is depicted by iterative 500 times of linear equation with every $K$. Figure 6(b) is derived from stroboscopic map model [4]. Figure 6(c) is the bifurcation diagram derived from EBM. Comparing three diagrams, the routes from bifurcation to chaos are similar. But the accurate positions of the first bifurcation point and output voltage are different. As shown in Figure 4, the bifurcation diagram in Figure 4(c) is closer to bifurcation diagram in Figure 4(a). Therefore, EBM has high accuracy in analyzing bifurcation and chaos phenomena.

Based on the above analysis, EBM still belongs to classification of stroboscopic map model although each modeling method is different. The disadvantage of EBM

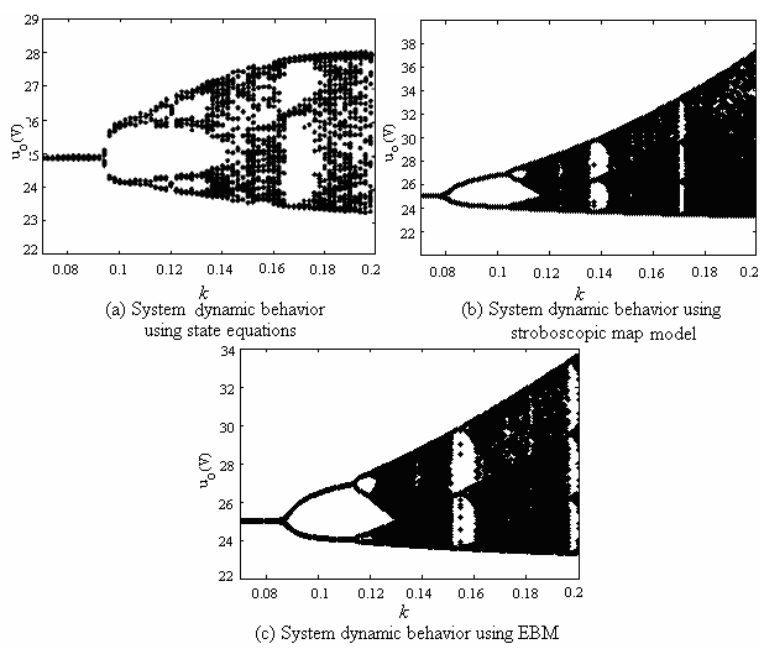

Figure 6. Bifurcation diagrams of output voltage simulated by three models

is that it can not analyze multiple pulses phenomena in one switching cycle. However, multiple pulses phenomena can be eliminated by a trigger which is added to PWM modulator (see Figure 1). Therefore, EBM is a unified model in analyzing nonlinear phenomena of converter.

\section{The Reason for Bifurcation and Chaos in Converters Based on Energy Balance View- point}

The mechanisms of bifurcation and chaos are so complex that there is not an unified criterion to identify them. The types of bifurcations are various, e.g., period-doubling bifurcation, saddle-node bifurcation, fork bifurcation, Hopf bifurcation and border collision bifurcation [5]. In particular, border collision bifurcation usually appears in piecewise smooth system. For normal bifurcation, the mechanism of bifurcation accords with bifurcation theory, i.e., bifurcation happens when eigenvalues of Jacobin matrix of switching map model traverse unit circle. Furthermore, bifurcation styles can be distinguished from traverse direction. However, border collision bifurcation can not be verified by eigenvalues. Border collision bifurcation will happen if some points on periodic orbits collide with the boundary. Many research results show that saturation of duty cycle in power converters results in border collision bifurcation. 
The general method for studying mechanisms of bifurcation is to seek breakthrough in state space. As shown in Figure 5, energy state of capacitor also exhibits bifurcation and chaos. Therefore, this paper firstly presents the mechanisms of bifurcation and chaos from energy balance viewpoint. From (9), we conclude that the present energy state is defined by former energy state and duty cycle. When duty cycle keeps steady, capacitor energy can keep balance in every switching period. But this is not the case. When capacitor energy can not keep balance in every switching period, switching time will increase to two switching cycles, four switching cycles, et al. Therefore, period 2 and period 4 bifurcations will happen. In general, imbalance of capacitor energy in one switching cycle results in bifurcation and chaos.

\section{Conclusions}

EBM still belongs to stroboscopic map model. But it has more accurate than stroboscopic map model. Comparing to iterative map model, the physical significance of EBM is more distinct. From energy locus diagrams, we can achieve abundant information about converters. Because energy balance theorem is universal rules in the world, EBM can be generalized to study the others nonlinear power converter as a unified model.

\section{REFERENCES}

[1] Middlebrook R D and Cuk S, "A general unified approach to modeling switching-converter power stage," IEEE Power Electron. Spec. Conf. Rec., pp. 18-34, 1976
[2] Mario di Bernardo and Francesco Vasca, "Discrete-time for the analysis of bifurcations and chaos in DC/DC converters," IEEE Transactions on Circuit System I , Vol. 47, No. 2, pp. 130-143, 2000 .

[3] Ma Xikui, Li Ming, Dai Dong, ZhaoHao, et al., "Reviews of research on complex behavior of power electronic circuits and systems," Transactions of China Electrotechnical Society, Vol. 21, No. 12, pp. 1-11, 2006.

[4] C. K. Tse, "Flip bifurcation and chaos in the three-state boost switching regulators," IEEE Transactions on Circuit System I , Vol. No. 1, pp. 16-23, 1994.

[5] C. K. Tse, Y. M. Lai, and H. H. C. Iu, "Hopf bifurcation and chaos in a free-running current-controlled Cuk switching regulator," IEEE Transactions on Circuit System I, Vol. 47, No. 4, pp. 448-457, 2000

[6] Guohui Yuan, Soumitro Banerjee, Edward Ott, et al., "Bordercollision bifurcations in the buck converter," IEEE Transactions on Circuit System I, Vol. 45, No. 7, pp. 707-716, 1998.

[7] H. H. C. Iu and C. K. Tse, "Bifurcation behavior in parallelconnected buck converters," IEEE Transactions on Circuit System I, Vol. 48, No. 2, pp. 233-240, 2001.

[8] Zhang Bo, Li Ping, and Qi Qun, "Method for analyzing and modeling bifurcation and chaos in DC/DC converters," Peoceedings of CSEE, Vol. 22, No. 11, pp. 81-86, 2002.

[9] Liu Weizeng, Zhang Hao, and Ma Xikui, "Analysis of intermittent bifurcations and chaos phenomena in boost PFC converters bystroboscopic map," Proceedings of CSEE, Vol. 25, No. 1, pp. 43-48, 2005

[10] Qu Ying and Zhang Bo, "The precise mathematical discrete model of buck converter in DCM and its analysis for bifurcation stability," Acta Electronica Sinica, Vol. 30, No. 8, pp. $1253-1256,2002$

[11] Ma Xikui, Liu Weizeng, and Zhang Hao, “Analysis of fast-scale bifurcations and chaos phenomena in boost PFC converter," Proceedings of CSEE, Vol. 25, No. 5, pp. 61-67, 2005. 